\title{
The effect of a strong external radiation field on protostellar envelopes in Orion
}

\author{
J. K. Jørgensen ${ }^{1,2 \star}$, D. Johnstone ${ }^{3,4}$, E. F. van Dishoeck ${ }^{1}$, and S. D. Doty ${ }^{5}$ \\ 1 Leiden Observatory, PO Box 9513, 2300 RA Leiden, The Netherlands \\ 2 Harvard-Smithsonian Center for Astrophysics, 60 Garden Street MS42, Cambridge, MA 02138, USA \\ e-mail: jjorgensen@cfa.harvard.edu \\ 3 National Research Council Canada, Herzberg Institute of Astrophysics, 5071 West Saanich Rd, Victoria, BC, V9E 2E7, Canada \\ 4 Department of Physics \& Astronomy, University of Victoria, Victoria, BC, V8P 1A1, Canada \\ 5 Department of Physics and Astronomy, Denison University, Granville, OH 43023, USA
}

Received 7 March 2005 / Accepted 5 December 2005

\section{ABSTRACT}

We discuss the effects of an enhanced interstellar radiation field (ISRF) on the observables of protostellar cores in the Orion cloud region. Dust radiative transfer is used to constrain the envelope physical structure by reproducing SCUBA $850 \mu \mathrm{m}$ emission. Previously reported ${ }^{13} \mathrm{CO}, \mathrm{C}^{17} \mathrm{O}$ and $\mathrm{H}_{2} \mathrm{CO}$ line observations are reproduced through detailed Monte Carlo line radiative transfer models. It is found that the ${ }^{13} \mathrm{CO}$ line emission is marginally optically thick and sensitive to the physical conditions in the outer envelope. An increased temperature in this region is needed in order to reproduce the ${ }^{13} \mathrm{CO}$ line strengths and it is suggested to be caused by a strong heating from the exterior, corresponding to an ISRF in Orion $10^{3}$ times stronger than the "standard" ISRF. The typical temperatures in the outer envelope are higher than the desorption temperature for $\mathrm{CO}$. The $\mathrm{C}^{17} \mathrm{O}$ emission is less sensitive to this increased temperature but rather traces the bulk envelope material. The data are only fit by a model where $\mathrm{CO}$ is depleted, except in the inner and outermost regions where the temperature increases above $30-40 \mathrm{~K}$. The fact that the temperatures do not drop below $\approx 25 \mathrm{~K}$ in any of the envelopes whereas a significant fraction of $\mathrm{CO}$ is frozen-out suggest that the interstellar radiation field has changed through the evolution of the cores. The $\mathrm{H}_{2} \mathrm{CO}$ lines are successfully reproduced in the model of an increased ISRF with constant abundances of $3-5 \times 10^{-10}$.

Key words. stars: formation - radiative transfer - astrochemistry

\section{Introduction}

Giant molecular clouds are the formation sites of massive stars in our Galaxy with the nearby Orion molecular clouds being the prime candidates for detailed studies of the earliest protostellar stages. An interesting difference for the studies of these cores compared to isolated systems is the large number of OB stars in the immediate vicinity: the UV radiation from these early-type stars ionizes their surrounding material within a few parsecs, and also affects the thermal balance and chemistry in intermediate and low-mass protostellar cores distributed over much larger scales. As recent studies indicate that large numbers of solar-type stars may be formed in these regions, it is of great importance to address the feedback between high- and lowmass star formation, in particular, for comparison with lowmass protostars formed in relative isolation in clouds such as Taurus.

In this paper we present a radiative transfer study of three intermediate mass protostars in Orion from the sample of Johnstone et al. (2003). The physical properties of their

\footnotetext{
* Current address: Harvard-Smithsonian Center for Astrophysics.
}

envelopes are established from $1 \mathrm{D}$ dust radiative transfer modeling, and $\mathrm{CO}$ isotopic and $\mathrm{H}_{2} \mathrm{CO}$ line observations are analyzed using Monte Carlo line radiative transfer. In particular, we discuss the importance of heating the protostellar envelope via an enhanced external radiation field and the constraints on this heating from optically thick CO isotopomers.

$\mathrm{Li}$ et al. (2003) recently estimated the gas kinetic temperatures for a sample of pre-stellar cores in Orion using inversion transitions of $\mathrm{NH}_{3}$. They found that these cores had lower temperatures than their surroundings, which they attributed to the impact of the strong interstellar radiation field (ISRF) in the Orion region. This is reminiscent of the situation for pre-stellar cores in more quiescent star forming regions where temperature gradients due to a standard interstellar radiation field are found in radiative transfer models reproducing submillimeter continuum maps (e.g., Evans et al. 2001). Wilson et al. (1999) suggested that the ratios of peak temperatures of optically thick lines with different critical densities could be used as a diagnostic of temperatures. Applied to cores in Orion their results also suggested strong temperature gradients in the region around Orion $\mathrm{BN} / \mathrm{KL}$ with more dense gas traced by $\mathrm{NH}_{3}$ inversion 
Table 1. Sample of sources in Orion. For details see Johnstone et al. (2003).

\begin{tabular}{lllll}
\hline \hline Source & & MMS6 & MMS9 & FIR4 \\
\hline$L_{\text {bol }}$ & {$\left[L_{\odot}\right]$} & 60 & 90 & 400 \\
Projected distance to the Trapezium $^{a}$ & {$[\mathrm{pc}]$} & 2.9 & 2.3 & 1.7 \\
$S_{850 \mu \mathrm{m}}($ peak $)$ & {$\left[\mathrm{Jy} \mathrm{beam}{ }^{-1}\right]$} & 7.5 & 2.5 & 7.5 \\
$I\left({ }^{13} \mathrm{CO} 3-2\right)^{b}$ & {$\left[\mathrm{~K} \mathrm{~km} \mathrm{~s}^{-1}\right]$} & 40.7 & 59.1 & 76.2 \\
$I\left(\mathrm{C}^{17} \mathrm{O} \mathrm{3}-2\right)^{b}$ & {$\left[\mathrm{~K} \mathrm{~km} \mathrm{~s}^{-1}\right]$} & 4.78 & 2.88 & 3.97 \\
$I\left(\mathrm{H}_{2} \mathrm{CO} 3_{03}-2_{02}\right)^{b}$ & {$\left[\mathrm{~K} \mathrm{~km} \mathrm{~s}^{-1}\right]$} & 5.33 & 3.07 & 9.82 \\
$I\left(\mathrm{H}_{2} \mathrm{CO} 3_{22}-2_{21}\right)^{b}$ & {$\left[\mathrm{~K} \mathrm{~km} \mathrm{~s}^{-1}\right]$} & 0.53 & 0.28 & 2.96 \\
$I\left(\mathrm{H}_{2} \mathrm{CO} 5_{05}-4_{04}\right)^{b}$ & {$\left[\mathrm{~K} \mathrm{~km} \mathrm{~s}^{-1}\right]$} & 2.56 & 0.94 & 5.24 \\
\hline
\end{tabular}

${ }^{a}$ Assuming a distance to Orion of 450 pc. ${ }^{b}$ Line intensity, $I=\int T_{\mathrm{MB}} \mathrm{d} v$.

lines colder $(\sim 24 \mathrm{~K})$ than less dense gas $(\sim 40 \mathrm{~K})$ probed by $\mathrm{CO}$ lines. In contrast Wilson et al. found similar temperatures probed by the $\mathrm{NH}_{3}$ and $\mathrm{CO}$ lines for cores further south from the Orion $\mathrm{BN} / \mathrm{KL}$ region and they argued that these cores are dominated by internal rather than external heating. This illustrates that an important point for the thermal balance of protostellar objects is whether external heating can compete with the central source luminosity.

The strong interstellar radiation field may also be reflected in the chemistry, for example by increasing the photodissociation and photoionization. The strength of the UV field can for example be probed by the emission of $\mathrm{C}^{+}$and has been applied to the Orion clouds where UV fields enhanced by factors of $10^{3}-10^{5}$ have been suggested (e.g., Tielens \& Hollenbach 1985a,b; Burton et al. 1990; Stacey et al. 1993; Mookerjea et al. 2003). The strong UV field will also lead to enhanced abundances of electrons and thus abundance decreases of molecular ions, such as $\mathrm{HCO}^{+}$and $\mathrm{N}_{2} \mathrm{H}^{+}$that otherwise work as destroyers of common molecules. Support for this is found within the OMC1 cloud core located immediately behind the Orion Nebula Cluster where Ungerechts et al. (1997) infer abundance gradients along the cloud, with increasing abundances of species such as $\mathrm{HCN}, \mathrm{CH}_{3} \mathrm{OH}, \mathrm{HC}_{3} \mathrm{~N}$, and $\mathrm{SO}$ toward the Orion $\mathrm{BN} / \mathrm{KL}$ region.

The astrochemistry study by Johnstone et al. (2003) considered a selection of cornerstone molecules in order to quantify the range of conditions for which individual molecular line tracers provide physical or chemical information. The morphological study compared a variety of locations along the Integral Shaped Filament (ISF) in Orion A (Bally et al. 1987; Johnstone \& Bally 1999), chosen to represent a range of physical conditions including enshrouded protostars, a bright PDR knot, and a shock front. The main conclusion of the paper was that the abundances of many molecular species correlate with source energetics, likely a result of the importance of temperature dependent desorption in maintaining gas-phase molecules. A significant finding of the study was the need for a warm outer envelope for all of the protostellar sources, as expected given the proximity of numerous $\mathrm{O}$ and $\mathrm{B}$ stars. The study suffered, however, from a simplistic treatment of the molecular line abundances derived from single temperature and density statistical equilibrium calculations and thus was unable to fully consider the effect of an enhanced radiation field on the outer regions of the embedded protostars.

This paper presents a continuation of the Johnstone et al. (2003) study, performing a detailed radiative transfer analysis of the physical and chemical properties of a subsample of the regions studied in that paper. We concentrate on the embedded intermediate mass protostellar sources, MMS6, MMS9, and FIR4 where the assumption of constant density and temperature throughout the envelope is most suspect. All three sources have large envelope masses, $M_{\text {env }}>10 M_{\odot}$. The implied dust temperatures, $T_{\mathrm{d}}$, for the sources are $>15 \mathrm{~K}$ while the ${ }^{13} \mathrm{CO}$ lines have peak brightness temperatures $\gtrsim 30 \mathrm{~K}$ which is difficult to produce unless the cores are bathed in an enhanced interstellar radiation field. The properties of the cores studied in this paper are summarized in Table 1 with further details presented in Johnstone et al. (2003). Even though the focus of this paper is on a few selected cores in Orion, a lot of the results brought up in this discussion are valid beyond these specific sources.

This paper is laid out as follows: Sect. 2 introduces the general problem, including the observational indications for a strong interstellar radiation field, and describes the modeling approach, including the possible constraints from continuum and line observations. Section 3 then discusses possible refinements and implications of the models, in particular, the resulting $\mathrm{CO}$ abundance structures and the reproduction of $\mathrm{H}_{2} \mathrm{CO}$ multi-transition observations. The evolutionary implications are discussed in Sect. 4. Section 5 concludes the paper suggesting possible further tests and future work.

\section{Models: constraining the ISRF}

Since the protostellar cores in Orion appear warmer than expected from heating by only the low luminosity central sources, they require the enhanced interstellar radiation field produced by the nearby OB stars for additional heating. In this section we present continuum and line radiative transfer models which describe the thermal structure of these cores and can be used for comparison to the observations. First, however, we describe direct evidence of the contribution from external heating of these cores - in particular from ${ }^{13} \mathrm{CO}$ isotopic lines and dust continuum observations obtained with the James Clerk Maxwell 


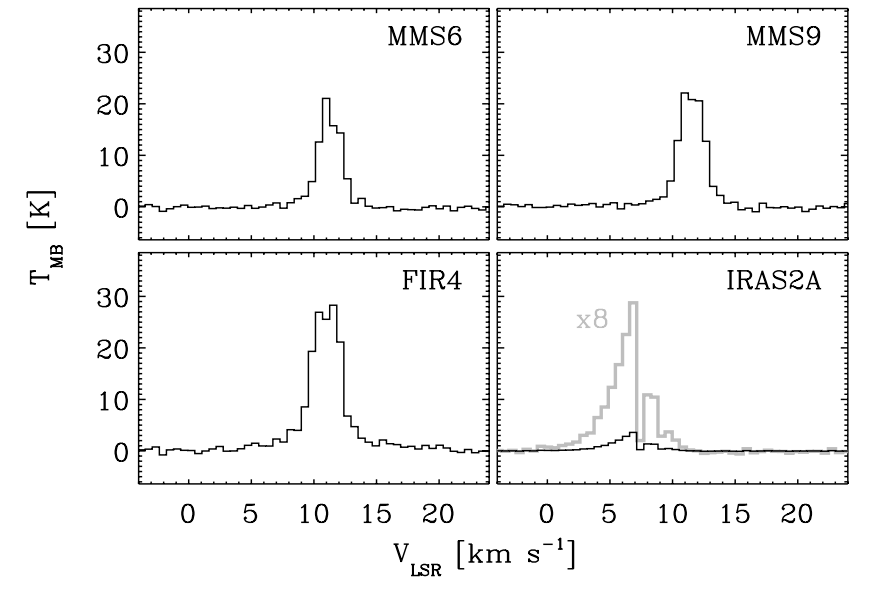

Fig. 1. JCMT ${ }^{13} \mathrm{CO} 3-2$ spectra from the three sources in Orion compared to NGC 1333-IRAS 2A.

Telescope in $\mathrm{a} \approx 15^{\prime \prime}$ beam (Johnstone et al. 2003; Jørgensen et al. 2004).

\section{1. ${ }^{13} \mathrm{CO} 3-2$ and ISRF}

Figure 1 compares the ${ }^{13} \mathrm{CO} \quad 3-2$ spectra toward the three sources. Also shown is the low-mass protostar, NGC 1333-IRAS 2A (Jørgensen et al. 2004) which has a lower luminosity $\left(16 L_{\odot}\right)$ than the Orion sources but also is located at a shorter distance $(220 \mathrm{pc})$. The peak temperatures for the three Orion sources are significantly higher than what is observed for NGC 1333-IRAS 2A. Also note that the lines are relatively Gaussian with less significant self-absorption. Since ${ }^{13} \mathrm{CO} 3-2$ is optically thick for the column densities representative of the studied cores, these spectra indicate that a relatively warm foreground layer must be present to prevent any self-absorption dip from the larger scale cold envelope. Comparing the ${ }^{13} \mathrm{CO}$ and $\mathrm{C}^{17} \mathrm{O} 3-2$ spectra from Johnstone et al. (2003) provides further evidence: the ${ }^{13} \mathrm{CO}$ lines are about a factor 2 broader than those of $\mathrm{C}^{17} \mathrm{O}$, suggesting that the lines are optically thick.

If the ${ }^{13} \mathrm{CO} 3-2$ line is optically thick, the peak temperature provides a measures of the excitation temperature in the region of the envelope where the optical depth reaches about unity. For the high densities in these envelopes the CO gas is in LTE (e.g., Jørgensen et al. 2002) and the excitation temperature directly measures the gas kinetic temperature. Furthermore at these densities the gas and dust temperatures are expected to be closely coupled (e.g., Ceccarelli et al. 1996; Doty \& Neufeld 1997) and can as a first approximation be assumed identical. In the following section we carry out the detailed line radiative transfer to calculate the full non-LTE line excitation and explore the relevance of the dust/gas temperature decoupling.

Correcting the peak brightness temperatures $T_{\text {peak }}=$ 17-28 K (Johnstone et al. 2003) for the Rayleigh-Jeans approximation, indicates gas temperatures of $T_{\mathrm{g}}=24-35 \mathrm{~K}$, assuming that the emission fills the beam. Such temperatures are, however, impossible to reconcile with the central star being the sole source of luminosity. For a spherically symmetric, optically thin envelope heated by a single central source of luminosity, the dust temperature distribution is approximated by:

$T_{\mathrm{d}}(r)=60\left(\frac{r}{2 \times 10^{17} \mathrm{~cm}}\right)^{-q}\left(\frac{L_{\mathrm{bol}}}{10^{5} L_{\odot}}\right)^{q / 2}$

where $q$ is related to the power-law index $\beta$ of the dust opacity law, $\kappa=\kappa_{0}\left(v / v_{0}\right)^{\beta}$, through $q=2 /(4+\beta)$, (Scoville \& Kwan 1976; Doty \& Leung 1994; Chandler et al. 1998; Chandler \& Richer 2000). This would be the temperature probed by the ${ }^{13} \mathrm{CO} 3-2$ lines if the emission comes from a shell with diameter larger than the JCMT beam which is very optically thick in these lines. To reach a specific temperature $T_{\mathrm{d}}$ at this radius the required luminosity is determined from Eq. (1):

$L_{\text {bol }}=6 \times 10^{5}\left(\frac{T_{\mathrm{d}}}{60 \mathrm{~K}}\right)^{2 / q}\left(\frac{d}{460 \mathrm{pc}}\right)^{2} L_{\odot}$.

Therefore, to produce peak temperatures $T_{\text {peak }}=17-28 \mathrm{~K}$ in the JCMT beam at $330 \mathrm{GHz}$ at the distance of Orion $(460 \mathrm{pc})$, central sources of $L \sim 6 \times 10^{3}-4 \times 10^{4} L_{\odot}$ are needed, significantly higher than the luminosity of the observed sources. In contrast, the $T_{\text {peak }} \approx 4 \mathrm{~K}$ for NGC 1333-IRAS 2A requires only 15-20 $L_{\odot}$, comparable to the bolometric luminosity of the source (Jørgensen et al. 2002). It therefore appears that the only way to explain the ${ }^{13} \mathrm{CO} 3-2$ line emission for the Orion sources is to introduce a strong external source of heating.

\subsection{Continuum models}

To establish the physical structure of each core we adopted the same approach as in Jørgensen et al. (2002), using the 1D radiative transfer code Dusty (Ivezić et al. 1999) to simulate $850 \mu \mathrm{m}$ SCUBA images and to constrain the density distribution of each core by comparing to observations. As in Jørgensen et al. the opacities for coagulated dust grains with thin ice mantles at a density of $10^{6} \mathrm{~cm}^{-3}$ from Ossenkopf \& Henning (1994) were adopted. The density profile is assumed to have a single radial power-law, $n=n_{0}\left(r / r_{0}\right)^{-p}$. The power-law index, $p$, is constrained through comparison between observed and modeled brightness profiles. The Orion protostars are located in the dense ridge of the ISF (Johnstone \& Bally 1999) complicating the extraction of a unique brightness profile. Greyscale images of each protostar are shown in Fig. 2. The hashed regions in each plot were not included in the circular averaging procedure used to define the brightness profiles. The flux at $850 \mu \mathrm{m}$ for each source from Johnstone et al. (2003) was used to constrain the overall dust content in the core, that is the optical depth at the fiducial wavelength (see, e.g., discussion in Jørgensen et al. 2002). Following Evans et al. (2001) we adopt the average ISRF from the solar neighborhood of Black (1994) with the UV field from Draine (1978) added and scale this at every wavelength with a constant factor, $\chi_{\mathrm{ISRF}}$ (i.e., $\chi_{\mathrm{ISRF}}=1$ for the standard ISRF).

Figure 3 illustrates the differences in the brightness profiles produced by varying the density profile slopes and the impact of the external interstellar radiation field. As shown, when including the ISRF a steeper density profile is required to fit the data. The best fit for MMS6 is a steep $r^{-2}$-envelope, whereas MMS9 and FIR4 have flatter $r^{-1.5}$-envelopes. 

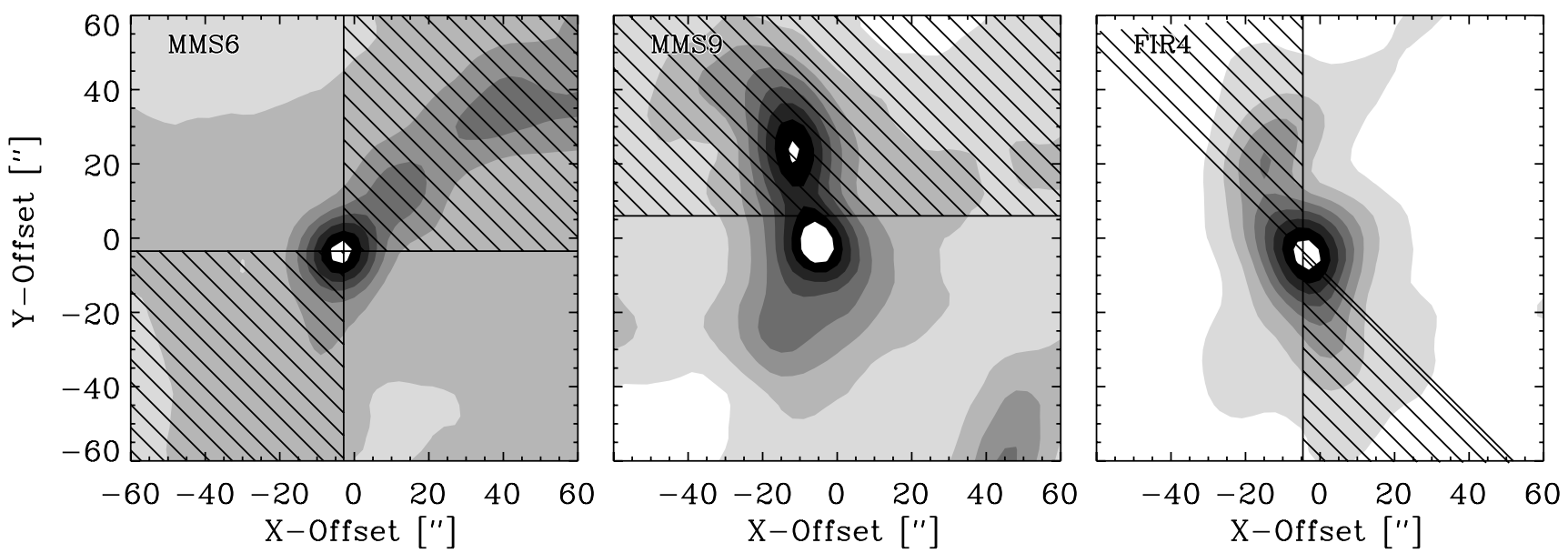

Fig. 2. SCUBA $850 \mu \mathrm{m}$ maps of the three Orion sources (from left to right: MMS6, MMS9 and FIR4). The regions which have been excluded when extracting the brightness profiles are hatched out.

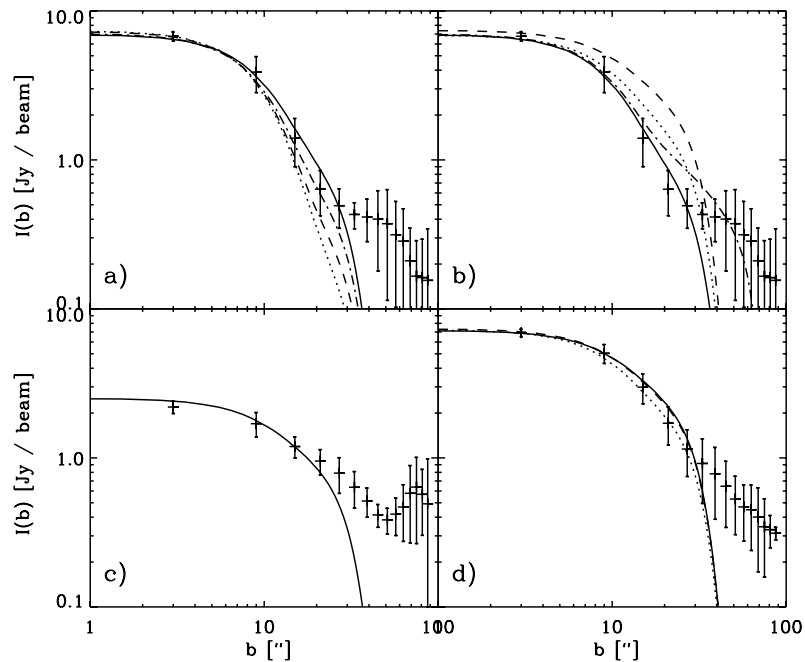

Fig. 3. Fits to the images of each of the Orion cores. a) MMS6 models with varying ISRF ( $\chi_{\text {ISRF }}$ of $1,10,100$ and 1000 with dotted, dashed, dotted-dashed and solid lines, respectively). b) MMS6 models with varying density slope ( $p=1.5,1.75,2.0$ with dashed, dotted and solid lines, respectively). The dashed-dotted line indicates a model with a density slope of 2.0 and an outer radius of $30000 \mathrm{AU}$ rather than 15000 AU. c) MMS9 model. d) FIR4 model with varying ISRF $\left(\chi_{\mathrm{ISRF}}=1000\right.$ and 10000 with dotted and solid lines, respectively).

Figure 4 compares the dust temperature profile for MMS6 with the JCMT beam size and the results for an optically thin envelope. The figure shows that a single pointing toward the source center will barely resolve the region where the dust temperature starts to increase due to the impact of the ISRF. This radius is therefore also very well-matched to the JCMT beam, which will selectively pick-up the outermost region. Since the envelope is optically thick, the temperature will be higher in the innermost region than predicted by the optically thin approximation, but decrease much more rapidly outside a few hundred AU. The luminosity derived from Eq. (2) above is therefore a lower limit to the required luminosity, further underscoring the need for a strong interstellar radiation field. Finally, it can be seen that the temperature does drop below $\approx 30 \mathrm{~K}$ in

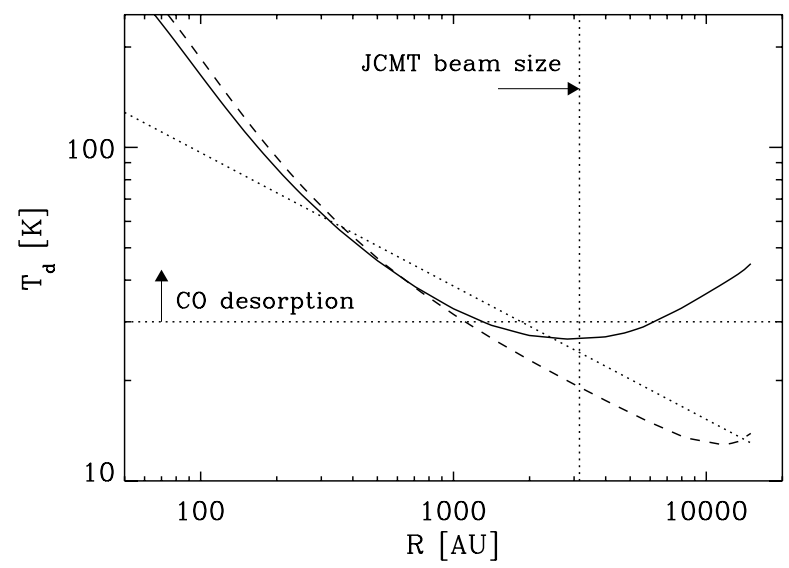

Fig. 4. Temperature profiles for MMS6: models for the enhanced $(\times 1000)$ and standard ISRF are indicated by the solid and dashed lines, respectively. The dotted line indicates the temperature profile for an optically thin envelope from Eq. (1) corresponding to $q=0.4$ (or $\kappa \propto \gamma^{\beta}$ with $\beta=1$ ). The size of the JCMT beam is indicated by the vertical dotted line, the $\mathrm{CO}$ evaporation temperature (see discussion in Sect. 3.1) with the horizontal dotted line.

the intermediate region at radii $\approx 1000-6000 \mathrm{AU}$, where $\mathrm{CO}$ depletion may occur. However, in the outermost region where the temperature increases again, $\mathrm{CO}$ will desorb rapidly from dust grains resulting in "standard" $\mathrm{CO}$ abundances.

Figure 5 compares the temperature profiles for varying strengths of the interstellar radiation field and internal luminosities. The figure clearly illustrates the competition between the interstellar radiation field and the internal source luminosity in defining the temperature structure - from the $L_{\text {int }}=100 L_{\odot}$ model with a standard interstellar radiation field where only the outer region ( $r>10000 \mathrm{AU}$ ) of the envelope is affected by the external heating to the $L_{\text {int }}=1 L_{\odot}$ model with $\chi_{\text {ISRF }}=1000$ where the interstellar radiation field dominates the temperature in to radii of $\sim 100 \mathrm{AU}$. For such a strong ISRF even a core without a central source or heating (or of very low luminosity) remains at high temperatures $\gtrsim 20 \mathrm{~K}$ all the way through to its center. Currently, the fluxes of these sources shortward 
Table 2. Summary of the tested models.

\begin{tabular}{|c|c|c|c|c|c|c|c|c|}
\hline & ISRF & $p$ & $\tau_{100}$ & $\begin{array}{l}n_{i} \\
{\left[10^{8} \mathrm{~cm}^{-3}\right]}\end{array}$ & $\begin{array}{l}\left\langle T_{\mathrm{d}}\right\rangle^{a} \\
{[\mathrm{~K}]}\end{array}$ & $\begin{array}{l}I\left({ }^{13} \mathrm{CO}\right)^{b} \\
{\left[\mathrm{~K} \mathrm{~km} \mathrm{~s}^{-1}\right]}\end{array}$ & $\begin{array}{l}I\left(\mathrm{C}^{17} \mathrm{O}\right)^{b} \\
{\left[\mathrm{~K} \mathrm{~km} \mathrm{~s}^{-1}\right]}\end{array}$ & In Figures \\
\hline \multirow[t]{9}{*}{ MMS6 } & 1 & 2.0 & 8.0 & 37 & 19 & $22.3(56)$ & $10.2(2.1)$ & 3a: dotted \\
\hline & 10 & 2.0 & 7.2 & 33 & 21 & $27.0(51)$ & $9.95(1.9)$ & 3a: dashed \\
\hline & 100 & 2.0 & 6.5 & 30 & 26 & $37.4(46)$ & $10.6(1.7)$ & 3a: dotted-dashed \\
\hline & 1000 & 1.5 & 2.4 & 5.9 & 31 & $58.6(37)$ & $17.4(1.4)$ & 3b: dashed \\
\hline & 1000-D & 1.5 & 2.4 & 5.9 & 31 & $55.4(37)$ & $17.2(1.4)$ & \\
\hline & 1000 & 1.75 & 3.9 & 13 & 32 & $56.2(39)$ & $14.2(1.5)$ & 3b: dotted \\
\hline & $1000^{*}$ & 2.0 & 5.6 & 26 & 36 & $53.6(40)$ & $11.1(1.5)$ & 3a,b: solid \\
\hline & $1000-2$ & 2.0 & 5.6 & 22 & 37 & $50.6(40)$ & $11.4(1.5)$ & 3b: dotted-dashed \\
\hline & 10000 & 2.0 & 4.5 & 21 & 54 & $73.4(28)$ & $10.2(1.1)$ & \\
\hline \multirow[t]{3}{*}{ MMS9 } & 1 & 1.5 & 1.1 & 2.7 & 17 & $34.6(12)$ & $8.30(0.47)$ & \\
\hline & $1000^{*}$ & 1.5 & 0.7 & 1.7 & 36 & $64.3(6.7)$ & $7.13(0.26)$ & 3c: solid \\
\hline & 10000 & 1.5 & 0.45 & 1.1 & 57 & $70.9(3.5)$ & $4.92(0.13)$ & \\
\hline \multirow[t]{4}{*}{ FIR4 } & 1 & 1.5 & 2.3 & 5.6 & 22 & $50.3(16)$ & $17.5(0.61)$ & \\
\hline & 1000 & 1.5 & 1.8 & 4.4 & 34 & $81.3(11)$ & $16.4(0.43)$ & 3d: dotted \\
\hline & 5000 & 1.5 & 1.5 & 3.7 & 45 & $99.2(8.5)$ & $14.7(0.32)$ & 3d: solid \\
\hline & $10000 *$ & 1.5 & 1.3 & 3.2 & 51 & $105.5(6.9)$ & $12.9(0.26)$ & \\
\hline
\end{tabular}

Notes: MMS6 model marked with "-D" is with gas and dust temperature decoupling taken into account. The inner and outer radii are 50 and 15000 AU, respectively except for the MMS6 model marked with "-2" which has an outer radius of 30000 AU. Models marked with "**” are the best fit models used in Table 3. Models for MMS6 have a constant turbulent broadening of $0.7 \mathrm{~km} \mathrm{~s}^{-1}$, those for MMS9 and FIR4 $1.0 \mathrm{~km} \mathrm{~s}{ }^{-1}$. ${ }^{a}$ Mass-weighted temperature: $\left\langle T_{\mathrm{d}}\right\rangle=\int T_{\mathrm{d}}(r) n(r) 4 \pi r^{2} \mathrm{~d} r / \int n(r) 4 \pi r^{2} \mathrm{~d} r{ }^{b}$ Modeled $J=3-2$ line intensity in $15^{\prime \prime}$ JCMT beam with optical thickness toward line and source center given in parentheses.

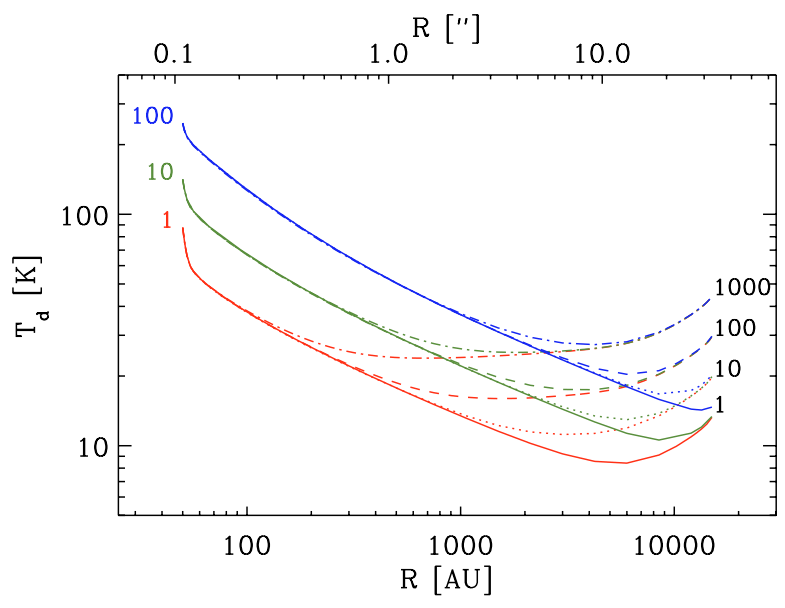

Fig. 5. Dust temperature profiles as function of radius (lower $X$-axis) and density (upper $X$-axis) for a $n \propto r^{-1.5}$-envelope with inner and outer radii of 50 and $15000 \mathrm{AU}$, respectively and $n_{0}=2.7 \times 10^{8} \mathrm{~cm}^{-3}$. Models are shown for luminosities of the inner source of 1,10 , and $100 L_{\odot}$ (red, green and blue lines) and $\chi_{\mathrm{ISRF}}$ of $1,10,100$ and 1000 (solid, dotted, dashed and dashed-dotted lines) as indicated in the left and right part of the figure, respectively.

of $100 \mu \mathrm{m}$ are not known and model fits have only been made to the long-wavelength tail of the SED (see Johnstone et al. 2003, and references therein for data). It is therefore not possible to separate the internal luminosity of the cores from the contribution from the external radiation field, and the temperature structure at the smallest radii therefore remains poorly constrained. Sensitive infrared observations, e.g., from the Spitzer Space Telescope, are needed to separate the contributions from the internal luminosities and the dust heated by the external radiation field to the total radiation emitted by the cores - such as previously done for giant molecular cloud cores near the galactic center based on ISO-LWS data by Lis et al. (2001).

Another problem is the determination of the outer radius of the cores. These cores are not formed in isolation but located in the Orion ridge, which is also picked up at larger scales of the brightness profiles as seen in Fig. 3. The ridge is found to have a density profile $n \propto r^{-2}$ on scales up to 60-300" (Johnstone \& Bally 1999). It would therefore seem more realistic to adopt an attenuated profile at the outer edge of $15000 \mathrm{AU}$ for the ISRF such as also suggested by the modeling by Shirley et al. (2005) and Evans et al. (2005). For a density profile $n \propto r^{-2}$ the column density from the outer edge, $r_{y}$, to infinity is $N=n\left(r_{y}\right) r_{y} \propto r_{y}^{-2} r_{y}$, i.e., declining as $r_{y}^{-1}$. For MMS6, for example, this implies a maximum extinction between the outer edge at $15000 \mathrm{AU}$ and the outside radiation field of $A_{\mathrm{V}} \approx 6$. If the outer radius of the cores is increased the resulting line intensities are unchanged, however (see Table 2): for the envelopes with density profile power-law slopes, $p$, of 1.5-2.0, the main contribution to the beam averaged column density is located at radii corresponding to the beam size or smaller. The ${ }^{13} \mathrm{CO}$ line becomes optically thick at roughly the same radius (and temperature) in the envelope.

\subsection{Lines}

Adopting the physical structure of each envelope from the dust models, the line emission is then modeled using the Monte Carlo radiative code of Schöier et al. (2002). This code was benchmarked together with other line radiative transfer 


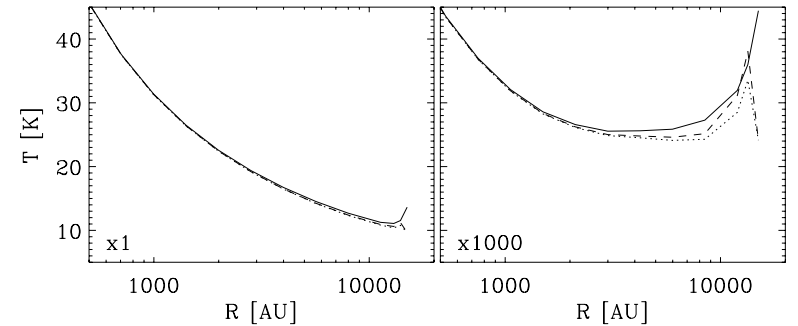

Fig. 6. Comparison between dust and gas temperature for a standard (left) and 1000× enhanced (right) interstellar radiation field. In both panels the solid line is the dust temperature. The gas temperature is indicated with the dashed line (assuming a turbulent broadening $1.6 \mathrm{~km} \mathrm{~s}^{-1}$ ) and the dotted line (assuming a turbulent broadening of $0.7 \mathrm{~km} \mathrm{~s}^{-1}$ ).

codes by van Zadelhoff et al. (2002). The molecular data were taken from the database of Schöier et al. (2005). For each model a turbulent broadening of $0.7-1.0 \mathrm{~km} \mathrm{~s}^{-1}$ was adopted, reproducing the observed line widths. For the optically thick ${ }^{13} \mathrm{CO}$ transitions inclusion of a more detailed velocity field may change the line opacity. On the other hand, if these objects indeed are young and the inside-out collapse model can be used to describe the velocity field or if the infall can be described by a power-law velocity field, the outermost regions should be close to static and the ${ }^{13} \mathrm{CO} 3-2$ lines dominated by turbulent broadening (see, e.g., discussion in Jørgensen et al. 2004).

To first order the gas and dust temperatures have been assumed to be perfectly coupled. This is justified by the high densities - generally $\gtrsim 10^{5} \mathrm{~cm}^{-3}$ for radii $\$ 7500 \mathrm{AU}$ - inferred throughout most of the envelope, which leads to strong thermal coupling between the gas and dust via collisions (Doty \& Neufeld 1997). In a strong external ISRF the dust temperature may be less closely coupled to the gas temperature due to photoelectric heating and UV pumping of $\mathrm{H}_{2}$. We tested the effect of the ISRF by calculating the gas temperature as described by Doty \& Neufeld (1997). The results are shown in Fig. 6. As can be seen, even in these strong external radiation fields, the densities studied here lead to dominant collisional coupling, and thus only marginal ( $\lessgtr$ a few $\mathrm{K}$ ) differences between the dust and gas temperatures.

For the first radiative transfer iteration, the abundances are assumed to be constant adopting a canonical $\mathrm{CO}$ abundance $X_{0}=1 \times 10^{-4}$ and ${ }^{12} \mathrm{C}:{ }^{13} \mathrm{C}$ and ${ }^{16} \mathrm{O}:{ }^{17} \mathrm{O}$ ratios of 70 and 1950 , respectively. The exact $\mathrm{CO}$ abundance will affect the derivation of the strength of the ISRF somewhat as it will regulate the location in the envelope where the observed ${ }^{13} \mathrm{CO}$ emission becomes optically thick. On the other hand, as the ${ }^{13} \mathrm{CO} 3-2$ emission does become optically thick the exact abundance structure in the interior of the envelope where depletion may occur does not affect the constraints on the ISRF (see also discussion below).

As shown in Fig. 7, an interstellar radiation field 1000 times stronger than the standard field is needed to account for the peak temperatures of the observed ${ }^{13} \mathrm{CO}$ spectra and fill out the self-absorption dip. It gives relatively Gaussian profiles and line widths of $2-2.5 \mathrm{~km} \mathrm{~s}^{-1}$ (FWHM) in agreement with observations. It is worth emphasizing here the differences between
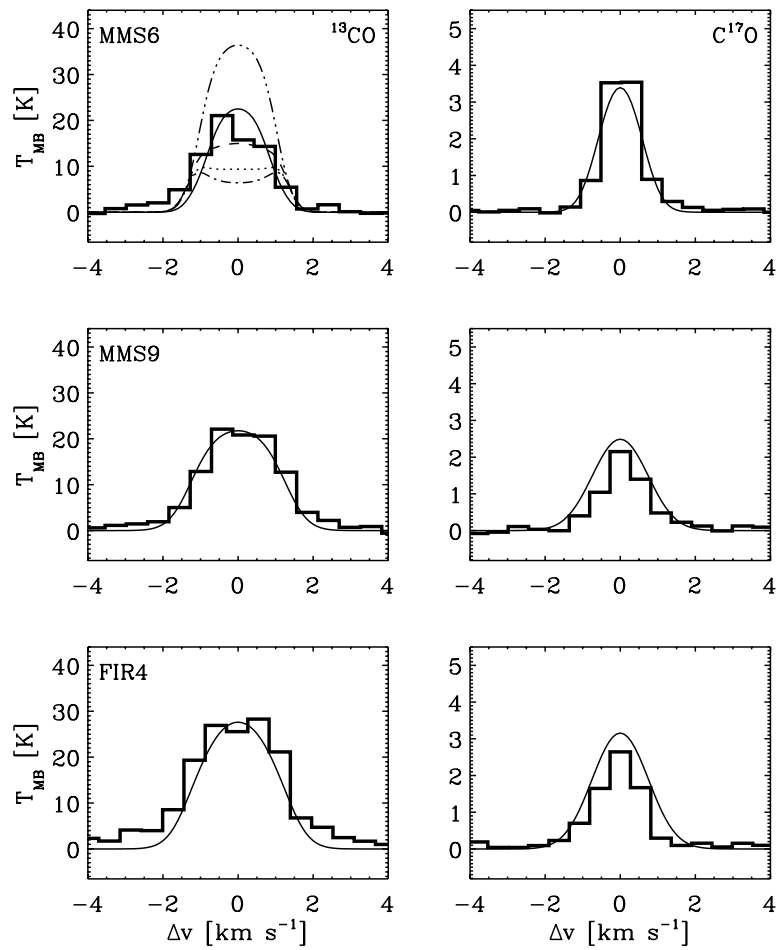

Fig. 7. Modeled and observed line profiles for ${ }^{13} \mathrm{CO}$ (left $)$ and $\mathrm{C}^{17} \mathrm{O}$ (right) $J=3-2$ for MMS6 (upper), MMS9 (middle) and FIR4 (lower). For MMS6 ${ }^{13} \mathrm{CO}$ models are shown for $1,10,100,10^{3}$ and $10^{4}$ times the standard ISRF with black dashed-dotted, dotted, dashed, solid and dashed- $3 \times$ dotted lines, respectively. For the remaining sources the best fit models from Tables 3 and 4 are shown.

Table 3. Models results for various CO abundance structures.

\begin{tabular}{lllll}
\hline \hline & ISRF & $\begin{array}{l}T_{\mathrm{de}} \\
{[\mathrm{K}]}\end{array}$ & $\begin{array}{l}I\left({ }^{13} \mathrm{CO}\right)^{b} \\
{\left[\mathrm{~K} \mathrm{~km} \mathrm{~s}^{-1}\right]}\end{array}$ & $\begin{array}{l}I\left(\mathrm{C}^{17} \mathrm{O}\right)^{b} \\
{\left[\mathrm{~K} \mathrm{~km} \mathrm{~s}^{-1}\right]}\end{array}$ \\
\hline MMS6 & 1000 & $\ldots$ & $53.6(40)$ & $11.1(1.5)$ \\
& $1000^{a}$ & 30 & $40.3(31)$ & $4.72(1.2)$ \\
& 1000 & 35 & $26.2(27)$ & $2.63(1.0)$ \\
MMS9 & 1000 & $\ldots$ & $64.3(6.7)$ & $7.13(0.26)$ \\
& $1000^{a}$ & 30 & $54.0(5.6)$ & $4.56(0.21)$ \\
& 1000 & 35 & $30.2(4.3)$ & $1.88(0.16)$ \\
FIR4 & 1000 & $\ldots$ & $81.3(11)$ & $16.4(0.43)$ \\
& 1000 & 30 & $71.0(9.3)$ & $10.6(0.35)$ \\
& 1000 & 35 & $48.1(7.4)$ & $5.68(0.28)$ \\
& $5000^{a}$ & 40 & $66.7(6.0)$ & $5.83(0.23)$ \\
& 10000 & 45 & $67.0(4.8)$ & $4.92(0.18)$ \\
\hline
\end{tabular}

For all models, a depleted abundance $X_{\mathrm{D}}$ has been used which is a factor 100 lower than the canonical CO abundance $X_{0}=10^{-4}$. ${ }^{a}$ Best fit model. ${ }^{b}$ See footnote $\mathrm{b}$, Table 2 .

the observations of the ${ }^{13} \mathrm{CO}$ and $\mathrm{C}^{17} \mathrm{O}$ lines: the $\mathrm{C}^{17} \mathrm{O}$ lines are significantly narrower $\left(1.0-1.5 \mathrm{~km} \mathrm{~s}^{-1}\right.$; FWHM) than the ${ }^{13}$ CO lines (Johnstone et al. 2003) (see Fig. 7). This simply reflects the fact that the $\mathrm{C}^{17} \mathrm{O}$ lines are optically thin and is confirmed by the models. Tables 2 and 3 summarize the results of the models for each source and Table 4 lists the parameters for the best fit model for each source. 
Table 4. Best fit parameters.

\begin{tabular}{llll}
\hline \hline Source & MMS6 & MMS9 & FIR4 \\
\hline$\chi_{\text {ISRF }}$ & $1 \times 10^{3}$ & $1 \times 10^{3}$ & $1 \times 10^{4}$ \\
CO depletion, $X_{0} / X_{\mathrm{D}}$ & 100 & 100 & 100 \\
Desorption temp., $T_{\mathrm{ev}}$ & $30 \mathrm{~K}$ & $30 \mathrm{~K}$ & $45 \mathrm{~K}$ \\
{$\left[\mathrm{p}-\mathrm{H}_{2} \mathrm{CO}\right]$} & $2.8 \times 10^{-10}$ & $3.6 \times 10^{-10}$ & $5.5 \times 10^{-10}$ \\
\hline
\end{tabular}

\section{Discussion}

In the previous section, the contribution from the external heating of the cores through the interstellar radiation field has been determined using the established framework of dust and gas radiative transfer. However, some simplifying assumptions were made and this section discusses how these models can be refined by taking the importance of chemistry into account.

\subsection{CO depletion and desorption temperature}

Jørgensen et al. (2002) derived the CO abundances for a sample of $\sim 20$ pre- and protostellar objects, among them NGC 1333-IRAS 2A. Clear signs of CO freeze-out were observed, with the highest degree of depletion found in the objects with the most massive envelopes. Models of the $\mathrm{C}^{17} \mathrm{O}$ lines suggest that freeze-out also occurs in the intermediate mass envelopes studied in this paper: adopting a constant canonical $\mathrm{CO}$ abundance of $10^{-4}$ as was done for ${ }^{13} \mathrm{CO}$ in the first iteration above, overproduces the observed $\mathrm{C}^{17} \mathrm{O} 3-2$ line intensities by about a factor three. It therefore seems that, at least in the region where the $\mathrm{C}^{17} \mathrm{O}$ lines are sensitive, the abundances must be lower. Since the $\mathrm{C}^{17} \mathrm{O} 3-2$ line generally is optically thin throughout the studied envelopes, the line is sensitive to the chemical structure in the interior of the envelope.

The freeze-out of $\mathrm{CO}$ - and thus its abundance - depends on temperature and density in the envelope and is therefore not constant but rather varies with radius. A CO desorption temperature higher than $20 \mathrm{~K}$ is suggested for low-mass protostellar cores from Monte Carlo modeling of multi-transition data and direct imaging through high-resolution interferometer observations. These observations show that $\mathrm{CO}$ evaporates off the dust grains in the innermost envelopes wherever the temperature becomes higher than 35-40 K (Jørgensen et al. 2002; Jørgensen 2004; Jørgensen et al. 2005a). Detailed chemical models of the low-mass protostar IRAS 16293-2422 by Doty et al. (2004) suggest a desorption temperature of CO of 20-40 K.

To simulate this effect, we introduce a simple piecewise constant "drop" abundance structure: we fix the abundances in three regions of the envelope, adopting a standard $\left[\mathrm{CO} / \mathrm{H}_{2}\right]$ abundance of $X_{0}=1 \times 10^{-4}$ in the inner and outermost region of the envelope where $\mathrm{CO}$ desorbs due to the temperature increasing above the $\mathrm{CO}$ desorption temperature $T \geq T_{\mathrm{ev}}$ and a depleted abundance $X_{\mathrm{D}}$ by two orders of magnitude in the region where the temperature drops below $T_{\mathrm{ev}}$. This model then only has one free parameter, $T_{\mathrm{ev}}$, which can be fit by comparison to the ${ }^{13} \mathrm{CO}$ and $\mathrm{C}^{17} \mathrm{O}$ line intensities. In fact, the observed transitions are not sensitive to the temperature, $T_{\mathrm{ev}}(\mathrm{in})$, at the innermost of these radii as the region inside this contributes only a negligible amount of material to the beam averaged $\mathrm{C}^{17} \mathrm{O}$ column density. Similar abundance profiles have been suggested for low-mass protostellar envelopes: in these envelopes the densities in the exterior are low so that the timescale for CO freeze-out is longer than the "age" of the core, which results in un-depleted abundances there. This is less of an issue for the Orion cores, however, since for these sources the densities are high enough at the edge that $\mathrm{CO}$ depletion does occur in the lifetime in the core, provided the temperature is low enough.

For MMS6 and MMS9 the ${ }^{13} \mathrm{CO}$ and $\mathrm{C}^{17} \mathrm{O}$ line intensities are well reproduced by these structures where the abundance decreases when (going from the inside) the temperatures decreases below $30 \mathrm{~K}$ at $\approx 1300 \mathrm{AU}$ (MMS6) and $\approx 2300 \mathrm{AU}$ (MMS9) and the abundance increases again when the temperature increases above $30 \mathrm{~K}$ (due to the external heating) at radii of $\approx 6400 \mathrm{AU}$ (MMS6) and $\approx 5300 \mathrm{AU}$ (MMS9) (Table 3). ${ }^{13} \mathrm{CO}$ still becomes optically thick in the outermost envelope and is thus not sensitive to the depletion region, whereas the $\mathrm{C}^{17} \mathrm{O}$ lines require an abundance decrease by 2 orders of magnitude. For both sources, models with $T_{\mathrm{ev}}=30 \mathrm{~K}$ are found to provide good fits to the data. A very interesting point is illustrated by the fits to FIR4: for this source it is not possible to fit the ${ }^{13} \mathrm{CO}$ and $\mathrm{C}^{17} \mathrm{O}$ simultaneously with $\chi_{\mathrm{ISRF}}=1000$ : for $T_{\mathrm{ev}}=30 \mathrm{~K}$ the $\mathrm{C}^{17} \mathrm{O}$ line is overproduced by a factor 2 by the envelope models whereas the ${ }^{13} \mathrm{CO} 3-2$ line is wellreproduced. Increasing the $\mathrm{CO}$ desorption temperature to $35 \mathrm{~K}$ (i.e., increasing the size of the $\mathrm{CO}$ depletion zone) lowers the $\mathrm{C}^{17} \mathrm{O}$ 3-2 line intensity to the observed value, but due to the ${ }^{13} \mathrm{CO} 3-2$ line being only moderately thick, the temperature probed by this line simultaneously drops and the line intensity correspondingly decreases by $35 \%$. If $\chi_{\mathrm{ISRF}}$ is increased by a factor 10 the envelope naturally becomes warmer, never dropping below $35 \mathrm{~K}$. However, for such a model both ${ }^{13} \mathrm{CO}$ and $\mathrm{C}^{17} \mathrm{O}$ line intensities are well-reproduced with a desorption temperature of $45 \mathrm{~K}$ (corresponding to radii of $1700 \mathrm{AU}$ and $7700 \mathrm{AU}$ ). This suggests a difference in the environment of FIR4 and the two other cores, with a stronger contribution of the ISRF impacting FIR4. Such a difference is in fact reasonable: FIR4 is located at about half the distance from the illuminating Trapezium stars compared to MMS6 (Table 1), so from pure geometric dilution an enhancement of the ISRF by about a factor of 4 is to be expected. An even larger enhancement difference between FIR4 and the two other sources is possible if the radiation field is attenuated by any intervening cloud material.

Rather than the ${ }^{13} \mathrm{CO}$ and $\mathrm{C}^{17} \mathrm{O}$ lines probing material with different levels of freeze-out, isotope selective photodissociation may result in changed abundances for the two species. As argued by van Dishoeck \& Black (1988), the photodissociation is dominated by line absorption (rather than continuum absorption) so that self-shielding and mutual shielding lines of more abundant isotopes and $\mathrm{H}_{2}$ are important for regulating the photodissociation rate of the various isotopes. This can in principle affect the abundance ratios of the isotopic species, e.g., by decreasing the less abundant species such as $\mathrm{C}^{17} \mathrm{O}$ relative to ${ }^{13} \mathrm{CO}$ compared to the "standard isotope ratios". Figure 8 shows the predicted $\mathrm{C}^{17} \mathrm{O} 3-2$ line intensities for models with 


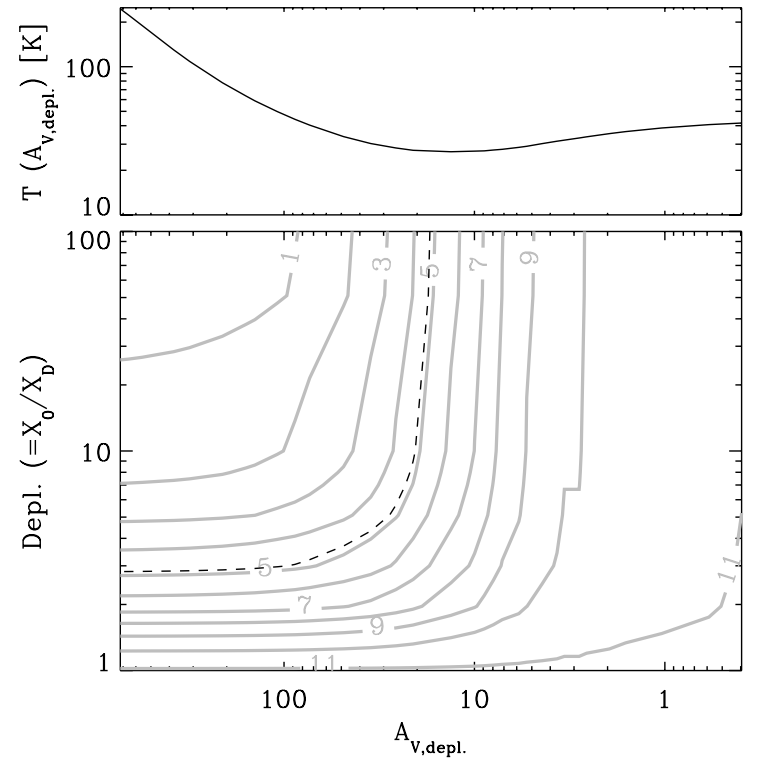

Fig. 8. Modeled line intensities in "jump models" for MMS6 where the $\mathrm{C}^{17} \mathrm{O}$ abundance is decreased to $X_{\mathrm{D}}$ from the outside inwards to a specific radius corresponding to an extinction $A_{\mathrm{V}, \text { depl. }}$ and a canonical abundance $X_{0}$ of $5.1 \times 10^{-8}$ (corresponding to a $\mathrm{CO}$ abundance of $10^{-4}$ ) within this radius. The density and temperature profiles are otherwise similar to the best fit model for MMS6. The contours are given in steps of $1 \mathrm{~K} \mathrm{~km} \mathrm{~s}^{-1}$ ranging from 1 to $11 \mathrm{~K} \mathrm{~km} \mathrm{~s}^{-1}$ as labeled. The dashed line indicates the observed line intensity for MMS6. The upper panel shows the temperature at the radius of the jump at $A_{\mathrm{V} \text {,depl. }}$.

a low $\mathrm{C}^{17} \mathrm{O}$ abundance from the outside of the envelope in to a radius corresponding to a specific column density (or extinction, $A_{\mathrm{V}, \text { depl. }}$ ) in the envelope simulating photodissociation of $\mathrm{C}^{17} \mathrm{O}$. Inside this radius the $\mathrm{C}^{17} \mathrm{O}$ abundance is increased to its canonical value assuming the standard isotope ratios. As shown the $\mathrm{C}^{17} \mathrm{O}$ abundance has to either be lower by one to two orders of magnitude into an $A_{\mathrm{V} \text {,depl }}$ of about 15-20 or lower by a factor of about 3 into an $A_{\mathrm{V}, \text { depl. }} \gtrsim 50$. Photodissocation will most likely only affect the outermost $\left(A_{\mathrm{V}} \lesssim\right.$ a few) envelope and only decrease the abundance by a few, it is therefore not sufficient to invoke isotope selective photodissociation to explain the differences between the material probed by ${ }^{13} \mathrm{CO}$ and $\mathrm{C}^{17} \mathrm{O}$.

\section{2. $\mathrm{H}_{2} \mathrm{CO}$ emission}

An interesting test of the derived models is how they account for the emission from other molecular species. In particular, ratios between a few key lines of $\mathrm{H}_{2} \mathrm{CO}$ have been suggested as a useful temperature probe (Mangum \& Wootten 1993) and may therefore be particularly sensitive to the impact of the ISRF. Johnstone et al. (2003) assumed constant densities and temperatures for each of the protostars in this paper and found temperatures of $<50 \mathrm{~K}$ for MMS6, $30 \mathrm{~K}$ for MMS9 and $80-90 \mathrm{~K}$ for FIR4. For comparison the mass-weighted temperatures,

$\left\langle T_{\mathrm{d}}\right\rangle=\int T_{\mathrm{d}}(r) n(r) 4 \pi r^{2} \mathrm{~d} r / \int n(r) 4 \pi r^{2} \mathrm{~d} r$

from the derived envelope models are $36 \mathrm{~K}$ for MMS6 and MMS9 and $51 \mathrm{~K}$ for FIR4 (see Table 2). The variations of these
Table 5. Summary of best-fit constant abundance $\mathrm{H}_{2} \mathrm{CO}$ models with differing contributions by the interstellar radiation field. All considered transitions are from para- $\mathrm{H}_{2} \mathrm{CO}$.

\begin{tabular}{llll}
\hline \hline Source & ISRF & Abundance $\left(\right.$ para- $\left.\mathrm{H}_{2} \mathrm{CO}\right)$ & $\chi_{\text {red }}^{2}$ \\
\hline MMS6 & 1 & $2.3 \times 10^{-10}$ & 5.2 \\
& $10^{3}$ & $2.8 \times 10^{-10}$ & 2.9 \\
MMS9 & 1 & $2.2 \times 10^{-10}$ & 5.1 \\
& $10^{3}$ & $3.6 \times 10^{-10}$ & 1.8 \\
FIR4 & 1 & $2.2 \times 10^{-10}$ & 6.7 \\
& $10^{4}$ & $5.5 \times 10^{-10}$ & 3.0 \\
NGC 1333-IRAS 2A & 0 & $3.9 \times 10^{-10}$ & $1.3^{b}$ \\
\hline
\end{tabular}

a $2 \sigma$ upper limit. ${ }^{b}$ For four fitted lines, including the $\mathrm{H}_{2} \mathrm{CO} 5_{24}-4_{23}$ transition at $363.946 \mathrm{GHz}$ (Jørgensen et al. 2005b).

temperatures between the studied sources therefore do appear to reflect the differences in the external heating, with FIR4 again being significantly warmer than the remaining sources.

The enhanced ISRF and thus more strongly varying temperature profile may, however, produce a discrepancy for sensitive temperature probes such as the ratios of the $\mathrm{H}_{2} \mathrm{CO}$ lines. To quantify this issue, simple constant abundance models were constrained by comparison to the observed $\mathrm{H}_{2} \mathrm{CO}$ line intensities. Table 5 lists the best fit models for models with both $\chi_{\mathrm{ISRF}}=1$ and the best fit, enhanced ISRF for each source. It is clear that the models with the enhanced ISRF provide significantly better fits to the observed $\mathrm{H}_{2} \mathrm{CO}$ line intensities. The abundances are found to be remarkably similar at $3-5 \times 10^{-10}$. For comparison Jørgensen et al. (2005b) found constant abundances of $3 \times 10^{-11}-4 \times 10^{-9}$ for a sample of 18 low-mass protostars.

The Orion cores are also interesting in the context of their $\mathrm{H}_{2} \mathrm{CO}$ abundance structures: enhanced $\mathrm{H}_{2} \mathrm{CO}$ abundances have been suggested for low-mass protostars in regions where ices evaporate from the dust grains at temperatures higher than 90-100 K although the evidence still is inconclusive (see discussions in Maret et al. 2004 and Jørgensen et al. 2005b). Since the cores in Orion have higher luminosities, the innermost hot $(T>90 \mathrm{~K})$ region is expected to be larger, and, despite their greater distances, these hot cores would be less diluted in the single-dish beam than the sources studied by Maret et al. (2004) and Jørgensen et al. (2005b) (diameters of 1.0-1.5" for the Orion sources compared to the $\lesssim 0.5^{\prime \prime}$ for the low-mass protostars). We find, however, that the observed $\mathrm{H}_{2} \mathrm{CO}$ lines are best fit by a constant abundance or a modest abundance jump at best. This conclusion is only based on a few lines, however, some of which may become optically thick and therefore more sensitive to the enhanced temperatures due to the external radiation field. Also, chemistry in the outer envelope, especially freeze-out, may affect the line intensities measured for $\mathrm{H}_{2} \mathrm{CO}$ (Schöier et al. 2004). Finally we reemphasize that it is not required that these sources have a luminous internal source from either the dust modeling or the gas modeling, which also is important in this context. Further studies of high excitation transitions at high angular resolution are needed to fully address these issues. 
The large spread in $\mathrm{H}_{2} \mathrm{CO}$ abundances for the low-mass protostellar cores (Jørgensen et al. 2005b) may be related to CO depletion: Maret et al. (2004) and Jørgensen et al. (2005b) find that the average abundances of $\mathrm{CO}$ and $\mathrm{H}_{2} \mathrm{CO}$ are correlated in the outer cold envelopes of low-mass protostars and high resolution interferometer observations indicate that $\mathrm{H}_{2} \mathrm{CO}$ follows a similar abundance structure as $\mathrm{CO}$ (Schöier et al. 2004). $\mathrm{H}_{2} \mathrm{CO}$ abundances for the three Orion sources were therefore also calculated with drop abundance structures based on the $\mathrm{CO}$ results, i.e., varying the undepleted and depleted $\mathrm{H}_{2} \mathrm{CO}$ abundances ( $X_{0}$ and $X_{\mathrm{D}}$, respectively) and adopting $T_{\mathrm{ev}}$ found for the fits to the $\mathrm{CO}$ lines. Figure 9 shows the $\chi^{2}$ confidence plots. Interestingly a drop in abundance is not indicated by the observed line intensities. Constant abundance or small increases in abundance within the $\mathrm{CO}$ depletion region are allowed by the $2 \sigma$ confidence levels for the observed sources.

\section{Evolutionary implications}

The points discussed above have interesting implications related to the evolution of the cores. The first concerns their history: as shown in Fig. 5 even for a pre-stellar core, i.e., a core with no internal source of heating, the temperature never decreases below $25 \mathrm{~K}$ in such an enhanced external radiation field considered in this paper. Under these conditions a given $\mathrm{CO}$ molecule would desorb again almost instantaneously after freeze-out so that only little depletion should be expected. It is, however, clear that $\mathrm{CO}$ is frozen-out in parts of the observed envelopes, suggesting that these regions must have been at lower temperatures earlier in the evolution of the cores.

An explanation may be that the strength of the UV field in the Orion region has changed significantly during the evolution of the studied cores. Plenty of circumstantial evidence suggests that the Trapezium cluster, which produces the majority of the local UV photons, is relatively young (O'Dell 2001). For example, Palla \& Stahler (2001) determine the age for $\Theta^{1}$ Ori B to be less than 100000 years. The most massive star in the Trapezium, $\Theta^{1}$ Ori $C$, shows considerable variability in its X-ray emission (Gagne et al. 1997) a likely indicator of extreme youth. Most, if not all of the low-mass stars in the Trapezium cluster are still proplyds, despite losing mass from their disks due to UV induced photoevaporation at rates of $10^{-7}$ to $10^{-6} M_{\odot} \mathrm{yr}^{-1}$ (Bally et al. 1998; Johnstone et al. 1998). Assuming that each disk has a minimum Solar nebula mass of $\approx 10^{-2} M_{\odot}$, this implies a lifetime of $<10^{5} \mathrm{yr}$ since the initiation of evaporation. Finally, the $\mathrm{H} \alpha+\mathrm{O}[\mathrm{III}]$ arcs observed around young low-mass stars near the high mass Trapezium stars trace out a wind driven bubble the size of which is very small, again suggesting extreme youth (O'Dell et al. 1997; Bally et al. 1998).

If the $\mathrm{CO}$ molecule is frozen-out at lower temperatures it may stay bound to the dust grains at the higher temperatures observed at present day, such as seen in low-mass protostellar envelopes where $\mathrm{CO}$ is expected to freeze-out in the cold $(T<15 \mathrm{~K})$ stages and evaporate as the protostar starts heating the envelope, increasing the temperature above $\approx 35-40 \mathrm{~K}$. One explanation for this according to laboratory experiments is that $\mathrm{CO}$ migrates into a porous ice when heated

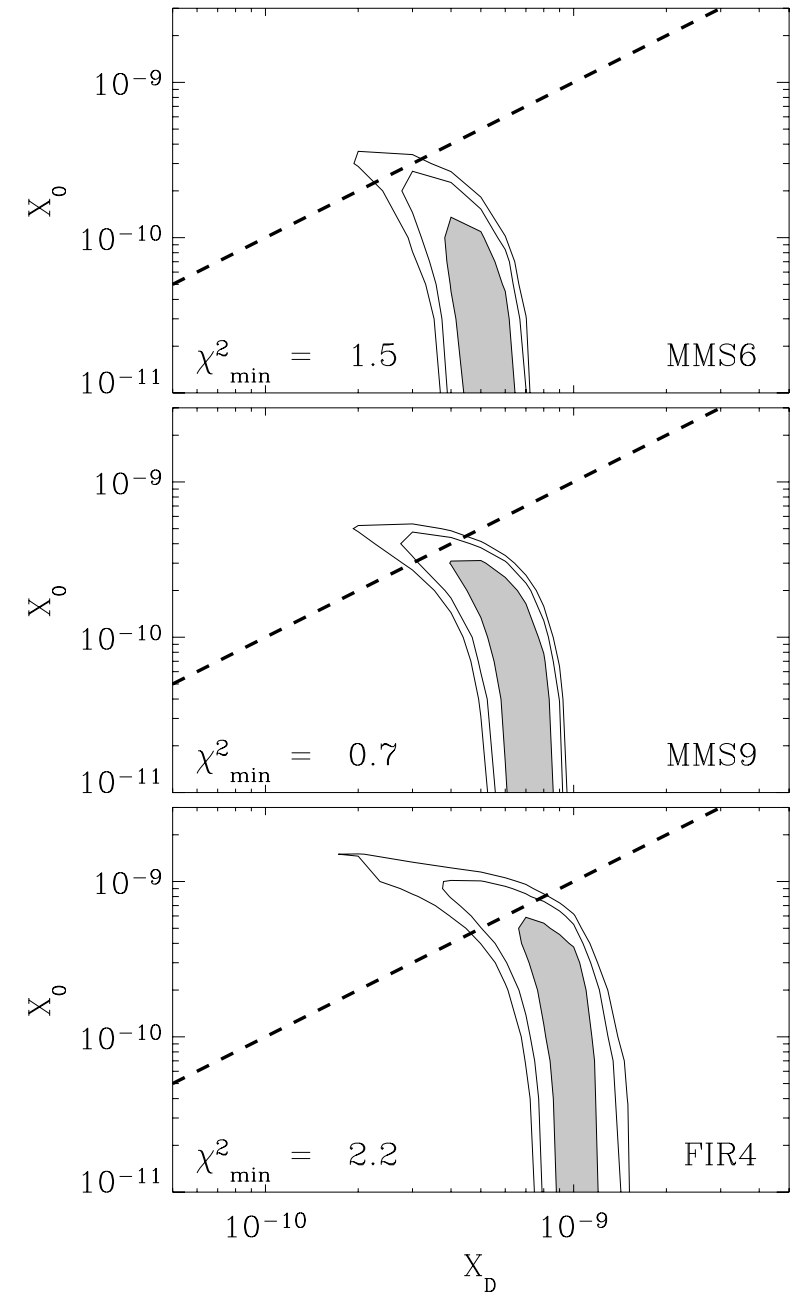

Fig. 9. $\chi^{2}$-confidence plots for fits to the $\mathrm{H}_{2} \mathrm{CO}$ lines toward MMS6, MMS9 and FIR4. The 68.3\% (" $1 \sigma$ "; also grey-scale), $90 \%$ and $95.4 \%$ (" $2 \sigma$ ") levels are shown. The abundance structures are assumed to follow those of the best fit $\mathrm{CO}$ models, i.e., with a change in abundance from the canonical value, $X_{0}$ to $X_{\mathrm{D}}$ in the intermediate regions where the temperatures drop below $30 \mathrm{~K}$ (MMS6 and MMS9) and $45 \mathrm{~K}$ (FIR4). The dashed lines indicate constant abundance models. The minimum $\chi_{\text {red }}^{2}$ for the models for each source is given in the lower left corner of each panel. Note that in all cases the best fit model has a higher $X_{\mathrm{D}}$ than $X_{0}$, but also that constant abundances are consistent with the data on the $2 \sigma$ level.

(Collings et al. 2003). It is therefore also not clear whether the determined values for $T_{\mathrm{ev}}$ in this paper correspond to the radii where the ice was formed at a lower temperature in an earlier stage or rather the radius where the temperature is high enough so that it evaporates at present. In fact the difference between FIR4 on one hand and MMS6 and MMS9 on the other may reflect such effects: Fig. 10 shows the evolution of the temperature distribution in a $100 L_{\odot}$ envelope with a similar density profile as in Fig. 5 but for varying ISRF strengths. At the earliest stages $\mathrm{CO}$ freezes out between radii where the temperature is lower than $21 \mathrm{~K}$, but as the ISRF strength increases the same radii will correspond to increasing temperatures. As the temperature then increases above $T_{\mathrm{ev}}, \mathrm{CO}$ desorbs and the radius for the outer edge of the depletion zone moves inwards. 

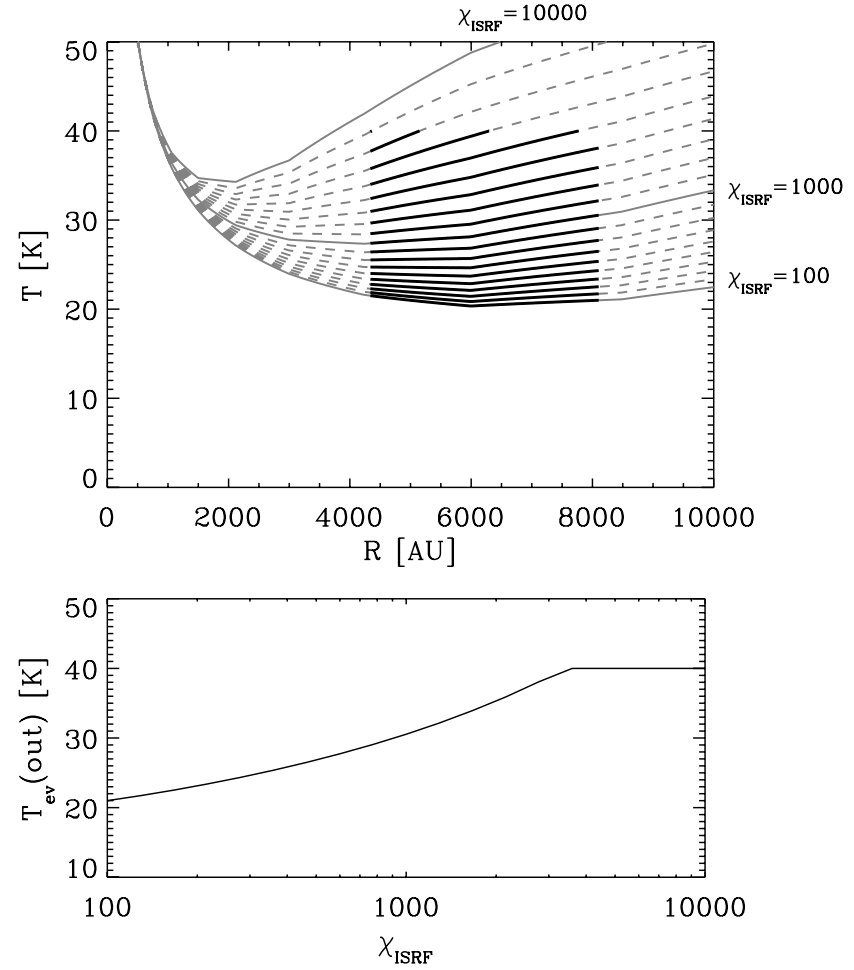

Fig. 10. The evolution of the temperature profiles (grey lines) for a $100 L_{\odot}$ core with density profile as in Fig. 5. Each line corresponds to increasing $\chi_{\mathrm{ISRF}}$ ranging from 100 to $10^{4}$ in logarithmic steps. The grey solid lines indicate the temperature profiles for $\chi_{\mathrm{ISRF}}=100,1000$ and 10000 . The thick black line in each plot indicates the radii where $\mathrm{CO}$ was frozen out for $\chi_{\mathrm{ISRF}}=100$. In the lower panel the evolution of the inferred $T_{\text {ev }}$ (out) with increasing ISRF strength is shown.

If this interpretation is true - the cores had CO freeze-out in the outer regions when formed, and then had $\mathrm{CO}$ evaporate when the OB stars "turned on" - this will also have important chemical implications in relation to the grain chemistry and for gas-phase species regulated by the amount of $\mathrm{CO}$. The $\mathrm{H}_{2} \mathrm{CO}$ and $\mathrm{CO}$ correlation can easily can be understood since the amount of $\mathrm{CO}$ and related $\mathrm{C}^{+}$and $\mathrm{O}$ is the main bottleneck for gas-phase $\mathrm{H}_{2} \mathrm{CO}$ production (Schöier et al. 2004). In a gas-phase chemical model using the approach of Doty et al. (2004), and adopting the physical conditions (incl. temperature and density) in MMS6, the $\mathrm{H}_{2} \mathrm{CO}$ abundances follows the $\mathrm{CO}$ abundance structure after $\sim 10^{5}$ years.

Although the abundance structures are similar in the Orion cores and in the low-mass protostellar envelopes, the grain chemistry might be significantly different. In both cases high abundances of, e.g., CO are expected in the outer envelopes, but whereas this is caused by low densities (and thus long timescales for freeze-out) in the low-mass protostellar envelopes, it is a result of direct desorption (high temperatures) in the Orion protostars. This has the consequence that the timescale where $\mathrm{CO}$ is available for surface chemical reactions e.g., hydrogenation forming $\mathrm{CH}_{3} \mathrm{OH}$ and the rates of these reactions are different in the Orion cores due to their dependence on temperature.

It will naturally be very interesting to test the importance of any photochemistry. In particular an increased $\mathrm{CO}$ abundance provides high amount of atomic carbon, which will lead to higher abundances of for example $\mathrm{CN}$ and $\mathrm{CS}$ in the outer envelopes relative to related species such as $\mathrm{HCN}$ and SO.

\section{Conclusions}

We have presented an analysis of submillimeter continuum and line observations toward three intermediate mass protostellar cores in the Orion molecular cloud using detailed radiative transfer models. The main conclusions of this study are:

- From simple luminosity and temperature considerations these sources must be subject to a strong external heating to reproduce observed ${ }^{13} \mathrm{CO} 3-2$ line temperatures and profiles.

- Detailed radiative transfer modeling confirms this, constraining the ISRF enhancement to $10^{3}-10^{4}$ times the standard ISRF from the studied sources. Different strengths of the ISRF between MMS6 and MMS9 in one case and FIR4 in the other can be explained in part due to the latter being closer to the Trapezium stars supplying the strong UV field.

- The $\mathrm{C}^{17} \mathrm{O}$ 3-2 lines are optically thin and thus not sensitive to the same temperature enhancement. Differences between the ${ }^{13} \mathrm{CO}$ and $\mathrm{C}^{17} \mathrm{O}$ line widths reflect this difference between the optically thick and thin lines, which is well reproduced by the models. To reproduce the $\mathrm{C}^{17} \mathrm{O}$ line intensities, significant $\mathrm{CO}$ depletion must have occurred in the part of the envelope where the temperature is lower than 30-45 K. A difference in the $\mathrm{CO}$ desorption temperatures derived for the sources, however, suggest an evolutionary difference with the OB stars (and thus UV field) evolving over similar timescales as the protostellar cores themselves.

- Multi-transition $\mathrm{H}_{2} \mathrm{CO}$ observations indicate high temperatures in the cores and their line ratios can only be reproduced in models with a strong ISRF. Constant abundances of $3 \times 10^{-10}-5 \times 10^{-10}$ provide a good fit to the line intensities.

This work illustrates the necessity of establishing the environmental impact for the evolution of pre- and protostellar cores for example in regions such as Orion where the impact of the radiation stars have a large impact on newly formed low- and intermediate mass protostars. As the above discussions also illustrate, detailed radiative transfer models can be used to address some of these issues, in particular if the chemistry is taken into account.

Additional mid-infrared observations of the cores can further constrain the relative contributions of the internal and external radiation field to the observed spectral energy distributions as it has previously been done for cores near the galactic center (Lis et al. 2001). Further continuum observations with (sub)millimeter interferometers such as the SMA, CARMA and eventually ALMA could further confirm the temperature structure of the envelopes at smaller scales and in particular whether desorption of molecules are seen toward their centers due to the heating by the central protostar. Likewise in an extreme interstellar radiation field such as that of the Orion further studies of the chemistry in the outer envelopes and intervening material in the ridge are interesting to fully understand 
the impact of the ionizing sources. Understanding these processes are important as the thermal balance and pressure of the cores controls the conditions and outcome of the collapse and thus establishing the properties of the newly formed stars.

Acknowledgements. We thank the referee, Neal Evans, for insightful comments which greatly improved the paper. The research of JKJ was funded through a Ph.D. stipend from the Netherlands Research School for Astronomy (NOVA) and NASA Origins Grant NAG513050. This research was also supported by a grant from the Natural Science and Engineering Research Council of Canada to DJ and a grant from The Research Corporation to SDD. Astrochemistry research in Leiden is supported by an NWO/Spinoza grant. DJ wishes to thank the Sterrewacht Leiden for its kind hospitality during the past four summers, during which much of this research was conducted.

\section{References}

Bally, J., Stark, A. A., Wilson, R. W., \& Langer, W. D. 1987, ApJ, 312, L45

Bally, J., Sutherland, R. S., Devine, D., \& Johnstone, D. 1998, AJ, 116, 293

Black, J. H. 1994, in ASP Conf. Ser. 58: The First Symposium on the Infrared Cirrus and Diffuse Interstellar Clouds, ed. R. M. Cutri, \& W. B. Latter

Burton, M. G., Hollenbach, D. J., \& Tielens, A. G. G. M. 1990, ApJ, 365,620

Ceccarelli, C., Hollenbach, D. J., \& Tielens, A. G. G. M. 1996, ApJ, 471,400

Chandler, C. J., Barsony, M., \& Moore, T. J. T. 1998, MNRAS, 299, 789

Chandler, C. J., \& Richer, J. S. 2000, ApJ, 530, 851

Collings, M. P., Dever, J. W., Fraser, H. J., McCoustra, M. R. S., \& Williams, D. A. 2003, ApJ, 583, 1058

Doty, S. D., \& Leung, C. M. 1994, ApJ, 424, 729

Doty, S. D., \& Neufeld, D. A. 1997, ApJ, 489, 122

Doty, S. D., Schöier, F. L., \& van Dishoeck, E. F. 2004, A\&A, 418, 1021

Draine, B. T. 1978, ApJS, 36, 595

Evans, N. J., Rawlings, J. M. C., Shirley, Y. L., \& Mundy, L. G. 2001, ApJ, 557, 193

Evans, N. J., Lee, J.-E., Rawlings, J. M. C., \& Choi, M. 2005, ApJ, 626, 919

Gagne, M., Caillault, J., Stauffer, J. R., \& Linsky, J. L. 1997, ApJ, 478, L87
Ivezić, Ž., Nenkova, M., \& Elitzur, M. 1999, User Manual for DUSTY, University of Kentucky Internal Report

Johnstone, D., \& Bally, J. 1999, ApJ, 510, L49

Johnstone, D., Hollenbach, D., \& Bally, J. 1998, ApJ, 499, 758

Johnstone, D., Boonman, A. M. S., \& van Dishoeck, E. F. 2003, A\&A, 412,157

Jørgensen, J. K. 2004, A\&A, 424, 589

Jørgensen, J. K., Schöier, F. L., \& van Dishoeck, E. F. 2002, A\&A, 389,908

Jørgensen, J. K., Schöier, F. L., \& van Dishoeck, E. F. 2004, A\&A, 416, 603

Jørgensen, J. K., Schöier, F. L., \& van Dishoeck, E. F. 2005a, A\&A, 435,177

Jørgensen, J. K., Schöier, F. L., \& van Dishoeck, E. F. 2005b, A\&A, 437, 501

Li, D., Goldsmith, P. F., \& Menten, K. 2003, ApJ, 587, 262

Lis, D. C., Serabyn, E., Zylka, R., \& Li, Y. 2001, ApJ, 550, 761

Mangum, J. G., \& Wootten, A. 1993, ApJS, 89, 123

Maret, S., Ceccarelli, C., Caux, E., et al. 2004, A\&A, 416, 577

Mookerjea, B., Ghosh, S. K., Kaneda, H., et al. 2003, A\&A, 404, 569

O'Dell, C. R. 2001, ARA\&A, 39, 99

O’Dell, C. R., Hartigan, P., Bally, J., \& Morse, J. A. 1997, AJ, 114, 2016

Ossenkopf, V., \& Henning, T. 1994, A\&A, 291, 943

Palla, F., \& Stahler, S. W. 2001, ApJ, 553, 299

Schöier, F. L., Jørgensen, J. K., van Dishoeck, E. F., \& Blake, G. A. 2002, A\&A, 390, 1001

Schöier, F. L., Jørgensen, J. K., van Dishoeck, E. F., \& Blake, G. A. 2004, A\&A, 418, 185

Schöier, F. L., van der Tak, F. F. S., van Dishoeck, E. F., \& Black, J. H. 2005, A\&A, 432, 369

Scoville, N. Z., \& Kwan, J. 1976, ApJ, 206, 718

Shirley, Y. L., Nordhaus, M. K., Grcevich, J. M., et al. 2005, ApJ, 632, 982

Stacey, G. J., Jaffe, D. T., Geis, N., et al. 1993, ApJ, 404, 219

Tielens, A. G. G. M., \& Hollenbach, D. 1985a, ApJ, 291, 747

Tielens, A. G. G. M., \& Hollenbach, D. 1985b, ApJ, 291, 722

Ungerechts, H., Bergin, E. A., Goldsmith, P. F., et al. 1997, ApJ, 482, 245

van Dishoeck, E. F., \& Black, J. H. 1988, ApJ, 334, 771

van Zadelhoff, G.-J., Dullemond, C. P., van der Tak, F. F. S., et al. 2002, A\&A, 395, 373

Wilson, T. L., Mauersberger, R., Gensheimer, P. D., Muders, D., \& Bieging, J. H. 1999, ApJ, 525, 343 